\title{
Refrigerant capacity of FeCrMoCuGaPCB amorphous alloys
}

\author{
V. Franco, J. M. Borrego, C. F. Conde, and A. Conde ${ }^{\text {a) }}$ \\ Departamento Física de la Materia Condensada, ICMSE-CSIC, Universidad de Sevilla, P.O. Box 1065, \\ 41080 Sevilla, Spain \\ M. Stoica \\ LTPCM-CNRS, Institut National Polytechnique de Grenoble, BP75, 38402 St. Martin d'Hères Campus, \\ 38031 Grenoble Cedex 1, France \\ S. Roth \\ Leibniz Institut für Metallische Werkstoffe, IFW Dresden, Postfach 270016, D01171 Dresden, Germany
}

(Received 25 May 2006; accepted 22 July 2006; published online 19 October 2006)

\begin{abstract}
The magnetocaloric effect of the FeCrMoCuGaPCB alloy series, suitable for being prepared as bulk amorphous alloys, has been studied. Optimal refrigeration cycles have a cold reservoir close to room temperature. The refrigerant capacity of these alloys is comparable to that of a Mo-containing Finemet-type alloy and is $\sim 40 \%$ bigger than that of other bulk amorphous alloys with comparable working temperatures. Analysis of the field dependence of the magnetic entropy change evidences a power law for all the magnetic regimes. (C) 2006 American Institute of Physics.
\end{abstract}

[DOI: 10.1063/1.2358311]

\section{INTRODUCTION}

The magnetocaloric effect ${ }^{1}$ (MCE) consists in the temperature change of a magnetic material upon the application of a magnetic field. When magnetized, the entropy of the spin subsystem is decreased and, under adiabatic conditions, the transfer of energy to the lattice provokes the heating of the material. Conversely, the adiabatic demagnetization of the material causes its cooling. Although this effect has been employed for reaching temperatures below $1 \mathrm{~K}$ since the second third of the last century, ${ }^{2}$ it was not until recently that it was successfully tested for near-room-temperature magnetic refrigeration. ${ }^{3}$ The reason for this long delay in extending the application temperature range is mainly associated with technical difficulties, although the search for more optimal materials would ease the improvement of the refrigerators. The commercial application of high temperature MCE refrigeration would bring increased energy efficiency and environmental benefits, which justify the intensive research efforts that are being undertaken. ${ }^{4-6}$

Ferromagnetic materials can be used for high temperature magnetic refrigeration because when the Curie temperature of the material is approached, the ferro-paramagnetic transition causes a peak in the magnetic entropy change associated with the magnetization/demagnetization of the material. As the Curie temperature of $\mathrm{Gd}$ is close to room temperature $(294 \mathrm{~K})$ and its magnetic moment is big, this element was the material of choice for developing the initial room temperature prototypes. ${ }^{3}$ The peak entropy change $\left(\left|\Delta S_{M}^{\mathrm{pk}}\right|\right)$ has been maximized with the discovery of the socalled giant ${ }^{7} \mathrm{MCE}$ and giant inverse MCE. ${ }^{8}$ Meanwhile, cost reduction is being investigated by using transition metal based alloys instead of rare earth based materials. ${ }^{9}$ In particular, there is a growing interest in studying the applicability of soft magnetic amorphous alloys as magnetic

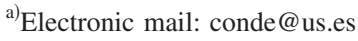

refrigerants ${ }^{10-15}$ due to their reduced magnetic hysteresis (virtually negligible), higher electrical resistivity (which would decrease eddy current losses), and tunable Curie temperature. In the case of bulk amorphous alloys, outstanding mechanical properties are also exhibited, but there are only a few studies in the literature concerning their MCE response. ${ }^{16,17}$ All the above mentioned characteristics of soft magnetic (bulk) amorphous alloys are beneficial for a successful application of the material.

Centering our attention in amorphous alloys that can be prepared in bulk form, it has recently been shown ${ }^{17}$ that the temperature corresponding to $\left|\Delta S_{M}^{\mathrm{pk}}\right|, T_{\mathrm{pk}}$, of the FeCoSiAlGaPCB alloy series can be tuned by changing the Fe/Co ratio in the alloy. However, although the increase in Co content shifts $T_{\mathrm{pk}}$ closer to room temperature, this compositional change also reduces the magnitude of the magnetocaloric effect (as evidenced by a decrease in both $\left|\Delta S_{M}^{\mathrm{pk}}\right|$ and refrigerant capacity of the material). An alternative method for tuning the magnetic transition temperature of an amorphous alloy consists in the addition of Mo and/or $\mathrm{Cr}^{18-20}$ In this work, the magnetocaloric effect of the FeCrMoCuGaPCB alloy series is analyzed. It will be shown that the refrigerant capacity of this series is comparable to that of a Mocontaining Finemet-type alloy with a similar Curie temperature, and is enhanced with respect to the FeCoSiAlGaPCB alloy series. These results, in combination with the large glass forming ability of the present series, make it a promising candidate for its application as high temperature magnetic refrigerant.

\section{EXPERIMENT}

Ribbons $(\sim 10 \mathrm{~mm}$ wide and $\sim 25 \mu \mathrm{m}$ thick $)$ of $\mathrm{Fe}_{65.5} \mathrm{Cr}_{4-x} \mathrm{Mo}_{4-y} \mathrm{Cu}_{x+y} \mathrm{Ga}_{4} \mathrm{P}_{12} \mathrm{C}_{5} \mathrm{~B}_{5.5} \quad(x, y=0,0.5,1 ; x+y$ $\leqslant 1$ ) were obtained by melt spinning. Transmission electron microscopy confirmed the amorphous character of the asquenched alloys with $x+y \leqslant 0.5$, but alloys with $x+y=1$ 


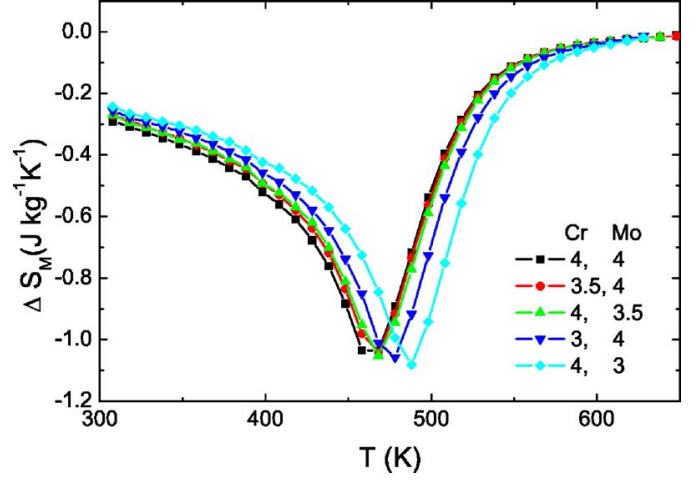

FIG. 1. (Color online) Temperature dependence of the magnetic entropy change for a maximum applied field of $1.5 \mathrm{~T}$. The lines are a guide to the eyes.

present a small density of bcc-Fe grains, with grain sizes in the $5-10 \mathrm{~nm}$ range and a crystalline volume fraction smaller than $5 \%$, not detectable by Mossbauer spectroscopy. ${ }^{20}$ The field dependence of magnetization was measured in a Lakeshore 7407 vibrating sample magnetometer using a maximum applied field $H=15 \mathrm{kOe}$ with field steps of $50 \mathrm{Oe}$, for constant temperatures in the range of 300-625 K with increments of $10 \mathrm{~K}$. Prior to the measurements, samples were stress relaxed by preannealing them at $675 \mathrm{~K}$.

The MCE can be characterized by the magnetic entropy change due to the application of a magnetic field $H$, which can be evaluated from the processing of the temperature and field dependent magnetization curves using a numerical approximation to the equation

$$
\Delta S_{M}=\int_{0}^{H}\left(\frac{\partial M}{\partial T}\right)_{H} d H
$$

where the partial derivative is replaced by finite differences and the integration is performed numerically.

In order to compare the performance of different materials, either the peak entropy change, $\left|\Delta S_{M}^{\mathrm{pk}}\right|$, or the refrigerant capacity (RC) are used. Whereas refrigeration at low temperatures requires a narrow temperature span of the refrigeration cycle, making $\left|\Delta S_{M}^{\mathrm{pk}}\right|$ the parameter of choice for comparing low temperature materials, high temperature refrigeration implies a wider temperature range and, consequently, RC is employed for comparison. According to Wood and Potter, ${ }^{21}$ the RC of a reversible refrigeration cycle operating between $T_{\text {hot }}$ and $T_{\text {cold }}$ (the temperatures of the hot and cold reservoirs, respectively) is defined as $\mathrm{RC}=\Delta S_{M} \Delta T$, where $\Delta S_{M}$ is the magnetic entropy change at the hot and cold ends of the cycle and $\Delta T=T_{\text {hot }}-T_{\text {cold. }}$. Moreover, hysteresis losses can be taken into account when evaluating the refrigerant material by subtracting them from the computed $\mathrm{RC},{ }^{22}$ making the comparison between materials with different coercivities more straightforward.

\section{RESULTS AND DISCUSSION}

Figure 1 shows the temperature dependence of the magnetic entropy change for the different alloys studied. All the samples present a similar behavior, with a peak at the Curie temperature of the amorphous alloy and a progressive fall to

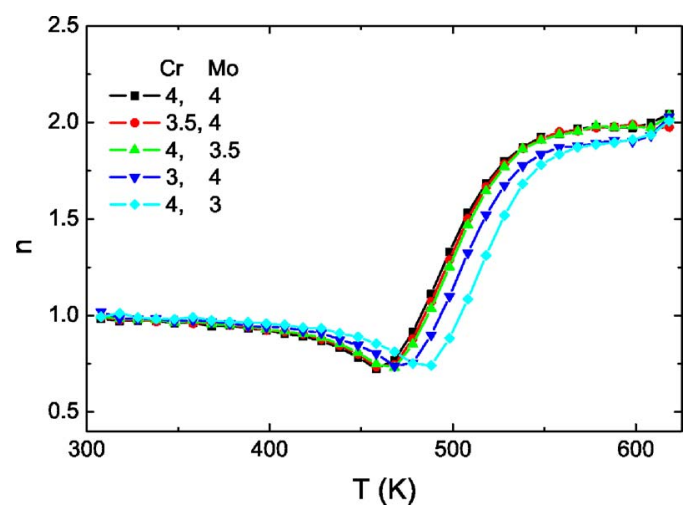

FIG. 2. (Color online) Temperature dependence of the local exponent $n$ for a maximum applied field of $1.5 \mathrm{~T}$. The lines are a guide to the eyes.

zero as the measuring temperature increases. The effect of nanocrystals embedded in the amorphous matrix would be to enhance the entropy change at higher temperatures. ${ }^{13}$ Even in the case that the nanocrystals behave as superparamagnetic particles (which is the expected situation for small crystalline volume fractions at temperatures above the Curie temperature of the amorphous matrix), ${ }^{23,24}$ their contribution to the magnetic entropy change would be an enhancement of the signal. ${ }^{25}$ Therefore, it is worth mentioning that the reduced crystalline fraction detected by transmission electron microscopy for the alloys with $x+y=1$ is not directly evidenced in these results, as MCE does reach zero for this temperature range.

The magnetic entropy change not only depends on the measuring temperature, but also on the value of the maximum applied field. The field dependence can be expressed as

$$
\Delta S_{M} \propto H^{n},
$$

where $n$ depends on the magnetic state of the sample. For ferromagnets above their Curie temperature, the direct integration of the Curie-Weiss law indicates that $n=2 .{ }^{4}$ However, for temperatures below the Curie temperature, no analytical expression is available. Moreover, the intrinsic irreversible behavior of ferromagnetic materials has to be taken into account in order to derive a thermodynamical model, ${ }^{26}$ a consideration which is especially important for materials with non negligible hysteresis (which is not the case in the present study). Nevertheless, on the basis of a mean field approach, the field dependence of the magnetic entropy change at the Curie temperature has been predicted to correspond to $n$ $=2 / 3{ }^{27}$ In order to determine the field dependence of the experimental $\left|\Delta S_{M}\right|$ data for the different magnetic regions of the studied samples, a local exponent ${ }^{16}$ can be calculated as

$$
n=\frac{d \ln \left|\Delta S_{M}\right|}{d \ln H} .
$$

Figure 2 shows the temperature dependence of the local exponent for the studied alloys, for a maximum applied field of $1.5 \mathrm{~T}$. The general features of the curves are similar to those recently found for Nanoperm-type alloys. ${ }^{15}$ In the ferromagnetic regime the local exponent is $n \approx 1$. At the Curie temperature of the amorphous alloy, a decrease in $n$ is observed. However, it does not reach $2 / 3$, as predicted by the 


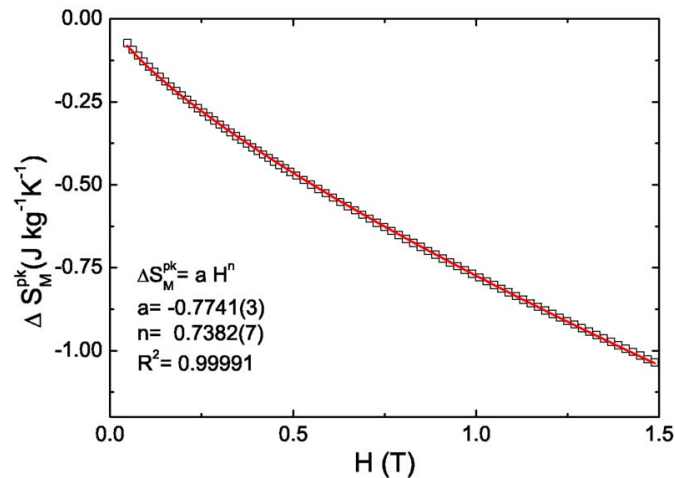

FIG. 3. (Color online) Field dependence of the peak entropy change for the $\mathrm{Cu}$-free alloy. The line is the fit to the data.

mean field approach, but decreases only to $\sim 0.73$. This value of the exponent, higher than the mean field predictions, could be due to local inhomogeneities in the amorphous alloy, causing a distribution of Curie temperatures, although the amorphous character of the alloy can also have an effect. On the other hand, discrepancies with mean field theories in the vicinity of a transition temperature can be expected. Above $T_{C}^{\mathrm{am}}$ the local exponent increases up to $n \approx 2$, as predicted by the Curie-Weiss law. The final increase in the exponent is ascribed to numerical errors, as the magnetic entropy change is negligible at these temperatures. It is worth noting that for the last two samples (those with $x+y=1$ ) the plateau in $n$ remains below that of the other three samples. This can be an effect of the small crystalline fraction detected by transmission electron microscopy, which distorts the Curie-Weiss behavior and reduces the value of the exponent. The field dependence of $\Delta S_{M}$ at $T_{\mathrm{pk}}$ for the $\mathrm{Cu}$-free alloy $(x=y=0)$ is also analyzed in Fig. 3 by representing $\Delta S_{M}^{\mathrm{pk}}$ versus field and fitting the curve to a power law. The value obtained for the exponent $(\sim 0.74)$ is comparable to the minimum in Fig. 2.

Figure 4 shows the refrigerant capacity of the alloys studied as a function of the temperature of the cold reservoir, $T_{\text {cold }}$, ranging from room temperature up to temperatures below $T_{\mathrm{pk}}$ of each alloy, i.e., below their Curie temperature (according to the above given definition of RC, for $T_{\text {cold }}$ $=T_{\mathrm{pk}}$ the refrigerant capacity is zero). The corresponding $T_{\mathrm{hot}}$

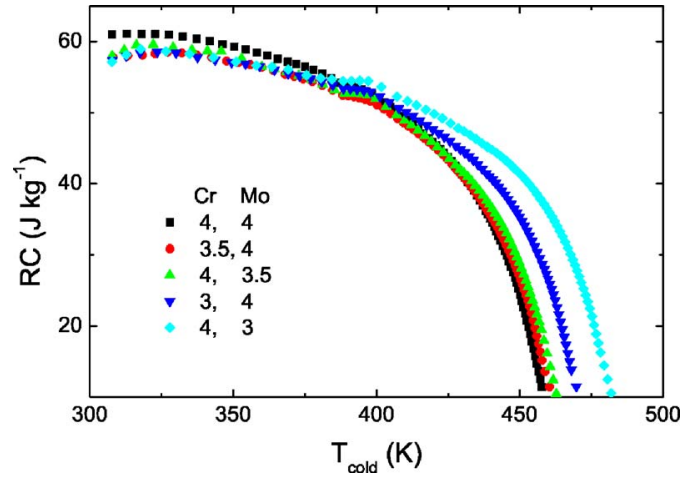

FIG. 4. (Color online) Dependence of the refrigerant capacity on the temperature of the cold end of the refrigeration cycle for all the studied alloys for a maximum applied field of $1.5 \mathrm{~T}$.

is selected by imposing $\Delta S_{M}\left(T_{\text {hot }}\right)=\Delta S_{M}\left(T_{\text {cold }}\right)$. As $T_{\text {cold }}$ separates from $T_{\mathrm{pk}}$, the refrigerant capacity of the material increases. An optimal refrigeration cycle can be found for all the alloys in the experimental temperature range, as evidenced by a maximum in RC. The maximum RC value obtained in this study is $61 \mathrm{~J} / \mathrm{kg}$ (Table I). This value is comparable to that of a Mo-containing Finemet-type alloy ${ }^{13}$ and is $\sim 38 \%$ bigger than that of an alloy suitable for being prepared as a bulk amorphous sample with a comparable Curie temperature, ${ }^{17}$ but remains well below that of a Nanopermtype alloy. ${ }^{15}$

Regarding the compositional effects evidenced in this study, there are no remarkable differences in the RC of the alloys studied, obtaining the bigger value for the $\mathrm{Cu}$-free sample. The comparison of $\left|\Delta S_{M}^{\mathrm{pk}}\right|$ indicates that the most favorable composition is the one where 1 at. \% Mo has been substituted by $\mathrm{Cu}$. In all cases, $\mathrm{Cu}$ substitution increases $\left|\Delta S_{M}^{\mathrm{pk}}\right|$, but this increase is always bigger when Mo is the substituted element. This behavior is in agreement with the bigger increase in the average hyperfine field obtained from Mossbauer spectroscopy when Mo is substituted by $\mathrm{Cu}^{20}$

\section{CONCLUSIONS}

The magnetocaloric effect of the FeCrMoCuGaPCB alloy series has been studied, as the outstanding mechanical

TABLE I. Peak entropy change, refrigerant capacity, temperatures of the cold and hot ends of the optimal refrigeration cycle, and temperature of the peak for a maximum applied field of $1.5 \mathrm{~T}$. Data for the last three alloys, measured under the same experimental conditions, are given for comparison.

\begin{tabular}{llllll}
\hline \hline & $\begin{array}{l}\left|\Delta S_{M}^{\mathrm{pk}}\right| \\
\left(\mathrm{J} \mathrm{kg}^{-1} \mathrm{~K}^{-1}\right)\end{array}$ & $\begin{array}{l}R C \\
\left(\mathrm{~J} \mathrm{~kg}^{-1}\right)\end{array}$ & $\begin{array}{l}T_{\text {cold }} \\
(\mathrm{K})\end{array}$ & $\begin{array}{l}T_{\text {hot }} \\
(\mathrm{K})\end{array}$ & $\begin{array}{c}T_{\text {pk }} \\
(\mathrm{K})\end{array}$ \\
\hline $\mathrm{Fe}_{65.5} \mathrm{Cr}_{4} \mathrm{Mo}_{4} \mathrm{Ga}_{4} \mathrm{P}_{12} \mathrm{C}_{5} \mathrm{~B}_{5.5}$ & 1.04 & 61 & 313 & 517 & 468 \\
$\mathrm{Fe}_{65.5} \mathrm{Cr}_{3.5} \mathrm{Mo}_{4} \mathrm{Cu}_{0.5} \mathrm{Ga}_{4} \mathrm{P}_{12} \mathrm{C}_{5} \mathrm{~B}_{5.5}$ & 1.04 & 59 & 328 & 517 & 468 \\
$\mathrm{Fe}_{65.5} \mathrm{Cr}_{4} \mathrm{Mo}_{3.5} \mathrm{Cu}_{0.5} \mathrm{Ga}_{4} \mathrm{P}_{12} \mathrm{C}_{5} \mathrm{~B}_{5.5}$ & 1.05 & 60 & 317 & 520 & 468 \\
$\mathrm{Fe}_{65.5} \mathrm{Cr}_{3} \mathrm{Mo}_{4} \mathrm{Cu}_{1} \mathrm{Ga}_{4} \mathrm{P}_{12} \mathrm{C}_{5} \mathrm{~B}_{5.5}$ & 1.06 & 59 & 325 & 527 & 478 \\
$\mathrm{Fe}_{65.5} \mathrm{Cr}_{4} \mathrm{Mo}_{3} \mathrm{Cu}_{1} \mathrm{Ga}_{4} \mathrm{P}_{12} \mathrm{C}_{5} \mathrm{~B}_{5.5}$ & 1.08 & 59 & 318 & 540 & 488 \\
$\mathrm{Fe}_{68.5} \mathrm{Mo}_{5} \mathrm{Si}_{13.5} \mathrm{~B}_{9} \mathrm{Cu}_{1} \mathrm{Nb}_{3}$ & 1.07 & 63 & 328 & 520 & 468 \\
$\left(\operatorname{Ref.~}_{13)}\right.$ & 1.60 & 82 & 325 & 531 & 488 \\
$\mathrm{Fe}_{78} \mathrm{Co}_{5} \mathrm{Zr}_{6} \mathrm{~B}_{10} \mathrm{Cu}_{1}$ & & & & & \\
$\left(\mathrm{Ref.}_{15}\right.$ & 0.93 & 44 & 308 & 550 & 498 \\
$\mathrm{Fe}_{29} \mathrm{Co}_{40} \mathrm{~B}_{9} \mathrm{C}_{2} \mathrm{Si}_{3} \mathrm{Al}_{5} \mathrm{Ga}_{2} \mathrm{P}_{10}$ & & & & & \\
$\left(\mathrm{Ref.}_{17}\right.$ & & & & & \\
\hline
\end{tabular}


properties of amorphous alloys with large supercooled liquid region can be beneficial for their application as magnetic refrigerants. The refrigerant capacity of this series is similar to that of a Mo-containing Finemet-type alloy and is $\sim 38 \%$ bigger than that of other bulk amorphous alloys with comparable working temperatures. The field dependence of the magnetic entropy change shows a power dependence for all the magnetic regimes of the samples, in agreement with previous results for Nanoperm-type amorphous alloys. In the ferromagnetic range the exponent is 1 . In the paramagnetic regime, well above the Curie temperature, the power is 2 , in agreement with the Curie-Weiss law. In the case of samples where quenched-in nuclei are present, the high-temperature exponent is reduced with respect to the Curie-Weiss predictions. The field dependence at the Curie temperature deviates from mean field predictions.

\section{ACKNOWLEDGMENTS}

This work was supported by the Spanish Government and EU-FEDER (Project MAT 2004-04618) and the PAI of Junta de Andalucía.

${ }^{1}$ E. Warburg, Ann. Phys. Chem. 13, 141 (1881).

${ }^{2}$ W. F. Giauque and G. P. MacDougall, Phys. Rev. 43, 768 (1933).

${ }^{3}$ C. A. Zimm, A. Jastrab, A. Sternberg, V. K. Pecharsky, K. A. Gschneidner, Jr., M. G. Osborne, and I. E. Anderson, Adv. Cryog. Eng. 43, 1759 (1998).

${ }^{4}$ A. M. Tishin, in Handbook of Magnetic Materials, edited by K. H. J. Buschow (Elsevier, Amsterdam, 1999), Vol. 12, pp. 395-524.

${ }^{5}$ K. A. Gschneidner, Jr. and V. K. Pecharsky, Annu. Rev. Mater. Sci. 30, 387 (2000).

${ }^{6}$ E. Brück, J. Phys. D 38, R381 (2005).
${ }^{7}$ V. K. Pecharsky and K. A. Gschneidner, Jr., Phys. Rev. Lett. 78, 4494 (1997).

${ }^{8}$ T. Krenke, E. Duman, M. Acet, E. F. Wassermann, X. Moya, L. Mañosa, and A. Planes, Nat. Mater. 4, 450 (2005)

${ }^{9}$ O. Tegus, E. Bruck, K. H. J. Buschow, and F. R. de Boer, Nature (London) 415, 150 (2002)

${ }^{10}$ D. Wang, K. Peng, B. Gu, Z. Han, S. Tang, W. Qin, and Y. Du, J. Alloys Compd. 358, 312 (2003).

${ }^{11}$ S. Atalay, H. Gencer, and V. S. Kolat, J. Non-Cryst. Solids 351, 2373 (2005).

${ }^{12}$ S. G. Min, K. S. Kim, S. C. Yu, H. S. Suh, and S. W. Lee, J. Appl. Phys. 97, 10M310 (2005).

${ }^{13}$ V. Franco, J. S. Blázquez, C. F. Conde, and A. Conde, Appl. Phys. Lett. 88, 042505 (2006).

${ }^{14}$ F. Johnson and R. D. Shull, J. Appl. Phys. 99, 08 K909 (2006).

${ }^{15}$ V. Franco, J. S. Blázquez, and A. Conde, J. Appl. Phys. 100, 064307 (2006).

${ }^{16}$ T. D. Shen, R. B. Schwarz, J. Y. Coulter, and J. D. Thompson, J. Appl. Phys. 91, 5240 (2002).

${ }^{17}$ V. Franco, J. M. Borrego, A. Conde, and S. Roth, Appl. Phys. Lett. 88, 132509 (2006)

${ }^{18}$ C. F. Conde, V. Franco, and A. Conde, Philos. Mag. B 76, 489 (1997).

${ }^{19}$ V. Franco, C. F. Conde, and A. Conde, J. Magn. Magn. Mater. 203, 60 (1999).

${ }^{20}$ J. M. Borrego, C. F. Conde, A. Conde, M. Stoica, S. Roth, and J. M. Greneche, J. Appl. Phys. 100, 043515 (2006).

${ }^{21}$ M. E. Wood and W. H. Potter, Cryogenics 25, 667 (1985).

${ }^{22}$ V. Provenzano, A. J. Shapiro, and R. D. Shull, Nature (London) 429, 853 (2004).

${ }^{23}$ V. Franco, L. F. Kiss, T. Kemény, I. Vincze, C. F. Conde, and A. Conde, Phys. Rev. B 66, 224418 (2002).

${ }^{24}$ V. Franco, C. F. Conde, A. Conde, and L. F. Kiss, Phys. Rev. B 72, 174424 (2005)

${ }^{25}$ R. D. McMichael, R. D. Shull, L. J. Swartzendruber, and L. H. Bennett, J. Magn. Magn. Mater. 111, 29 (1992).

${ }^{26}$ V. Basso, G. Bertotti, M. LoBue, and C. P. Sasso, J. Magn. Magn. Mater. 290, 654 (2005).

${ }^{27}$ H. Oesterreicher and F. T. Parker, J. Appl. Phys. 55, 4334 (1984). 